\title{
Dental caries and first permanent molar pit and fissure morphology in 7- to 8-year-old children in Wuhan, China
}

\author{
Jin-Dong Wang ${ }^{1,2}, \mathrm{Xi}_{\text {Chen }}{ }^{1}$, Jo Frencken ${ }^{3}$, Min-Quan Du ${ }^{1}$ and Zhi Chen ${ }^{1}$
}

To obtain the caries experience and, plaque accumulation severity and pit and fissure morphology in first permanent molars in 7-8 children in Wuhan, as a reasonable prediction of caries risk and preventive attention in the future, a convenient sample of five primary schools in the vicinity of the Wuhan University School and Hospital of Stomatology was drawn. Two calibrated examiners orally examined all present grade 2 children in the classroom, using standard caries plaque and tooth morphology criteria. Dental caries was scored at enamel $\left(D_{2}\right)$ and dentine $\left(D_{3}\right)$ for tooth and surface level. Independent variables were age, gender and school. Data analysis used analysis of variance and $t$-test. The sample comprised 1043 7- and 8-year-olds. The prevalence of dental caries in permanent dentition was $8.7 \%$ and in primary dentition, $68.7 \%$. Mean Decayed, Missing, Filled Teeth/S (DMFT/S) scores were 0.11 and 0.14 , respectively. Mean dmft/s scores were 2.8 and 5.0 . The $\mathrm{d}$-component constituted $75 \%$ of the $\mathrm{d}_{3} \mathrm{mft}$ index, while enamel carious lesions constituted $36 \%$ of the total number of carious lesions ( $d_{2,3}$-component). Prevalence of medium and deep pits and fissures was $84.6 \%$. Prevalence of medium and severe plaque accumulation was $67.4 \%$. Prevalence of dental caries in the deciduous and permanent dentitions of 7- to 8-year-old children was high. Deep pits and fissures in high caries risk children should be sealed. International Journal of Oral Science (2012) 4, 157-160; doi:10.1038/ijos.2012.34; published online 15 June 2012

Keywords: dental caries; oral epidemiology; plaque; tooth morphology

\section{INTRODUCTION}

The tremendous rise in the economy of China over the last decade has increased the possibility that the number of children with high caries risk may also have risen. This assumption was investigated to elicit cohort effects, using a mixed-longitudinal study design in conjunction with the Age-Period-Cohort analysis. ${ }^{1-2}$ In permanent dentitions, an increase in carious lesion development would manifest itself first in pits and fissures of first molars. ${ }^{3}$ Such tooth surfaces would need to be sealed, which is considered as the most cost-effective way. ${ }^{4-8}$

Two national oral health surveys among 5- and 12-year-old Chinese children have been carried out since $1995 .{ }^{9-10}$ However, these surveys excluded assessment of carious lesion development in newly erupted first permanent molars. Few studies done in China have assessed the caries status of newly erupted first molars. ${ }^{11-12}$ None were longitudinal in nature. Therefore, assessment using the mixed-longitudinal study design was needed regarding the caries status in children at the ages of first permanent molar eruption, in order to assess a cohort effect in young Chinese children. Furthermore, the research was needed for the inclusion of children with pits and fissures in first permanent molars at risk of developing carious lesions in a currently ongoing in vivo sealant study.

Therefore, the aim of this paper is to report on the caries experience in the dentition of 7- to 8-year-old children, plaque accumulation and pit and fissure morphology in first permanent molars in young Chinese children.

\section{MATERIALS AND METHODS}

\section{Sampling procedure}

This epidemiological research preceded an intervention study on the effectiveness of sealants. On the basis of a power calculation for determination of the required sample size of the intervention study, 1000 grade 2 children needed to be examined in the epidemiological study. To obtain the required sample size, five primary schools, conveniently situated in the neighborhood of Wuhan University School and Hospital of Stomatology in Wuhan, China, were chosen. This facilitated easy transport of equipment and personnel to and from the schools. One of the schools was already participating in a longstanding cooperation program with the Dental School, based on providing twice-yearly oral health education covering preventive activities such as fluoride mouth rinsing. The sample area was inhabited by families from various socioeconomic backgrounds.

Wuhan, the capital city of Hubei Province, has a population of about eight million people and its GDP increased from \$89 in 1978 to $\$ 3960$ in 2008. The study was accepted by the ethical committee of Wuhan University (Reference No. 200704).

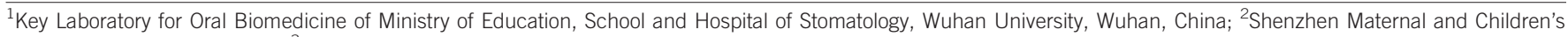
Hospital, Shenzhen, China and ${ }^{3}$ Department of Global Oral Health, Radboud University Nijmegen Medical Centre, College of Dental Sciences, Nijmegen, The Netherlands Correspondence: Professor Z Chen; Professor MQ Du, Key Laboratory for Oral Biomedicine of Ministry of Education, School and Hospital of Stomatology, Wuhan University, Wuhan 430072, China 
Table 1 Dental caries diagnostic index used in the present study

\begin{tabular}{lll}
\hline \multicolumn{2}{c}{ Code } & \\
\hline Permanent & Deciduous & Description \\
\hline 0 & A & Sound surface \\
1 & B & $\begin{array}{l}\text { Early enamel lesion. White/opaque or brownish/dark } \\
\text { lesion in enamel only, including loss of tooth surface, } \\
\end{array}$ \\
& Considered being active or inactive \\
2 & Carious lesion involving the dentine slightly; lesion cannot \\
& D & De pentinal lesion; lesion can be penetrated with CPI probe \\
3 & E & Dentinal lesion: pulp possibly or definitely exposed \\
5 & G & Restoration \\
6 & H & Sealant \\
7 & 8 & Missing due to caries \\
9 & 9 & Unerupted permanent tooth \\
\hline
\end{tabular}

$\mathrm{CPI}$, community periodontal index.

Table 2 Pits and fissure morphology index ${ }^{14}$ used

\begin{tabular}{lll}
\hline Score & Categories & Description \\
\hline 1 & Shallow & $\begin{array}{l}\text { Cuspal inclines meet at a wide angle. The base of the fissure is } \\
\text { visible. No visible clefting between the cuspal inclines }\end{array}$ \\
3 & Intermediate & $\begin{array}{l}\text { Cuspal inclines meet at an angle narrower than the designated } \\
\text { shallow fissures. The base of the fissure is usually visible. } \\
\text { Clefts are visible at a uniform width of the fissure }\end{array}$ \\
& $\begin{array}{l}\text { Cuspal inclines meet at a narrow angle. The vase of the fissure } \\
\text { is not visible. Slit-like clefting is visible between the cuspal } \\
\text { inclines }\end{array}$ \\
\hline
\end{tabular}

Oral examination

All grade 2 students present on a day in December 2007 were examined orally on the school premises. A mouth mirror and explorer were used. Each child lay on a table while examined. The oral cavity was illuminated by a removable site light providing adequate light to enable the two seated and calibrated examiners to diagnose dental caries, assessing plaque accumulation and first molar pit and fissure morphology. No X-rays were taken. Two trained chair-side assistants did the recording.

Plaque was assessed on the six index teeth according to the Greene and Vermillion index. ${ }^{13}$ The criteria described in Table 1 were used in diagnosing carious lesions. Plaque and/or debris were removed from the tooth surfaces with an explorer if necessary, to facilitate proper carious lesion diagnosis. The community periodontal index (CPI) probe was used for diagnosing small dentine carious lesions suitable for treatment with the atraumatic restorative treatment approach (Table 1). The pit and fissure morphology of first molars was assessed according to the criteria described by Symons et al. ${ }^{14}$ (Table 2).

\section{Reliability}

A calibration exercise was carried out prior to the start of the study, under the supervision of an experienced epidemiologist. Further assurance of the quality of the data was obtained, as the examiners discussed the clinical data of 20 children during the first two examination days. The kappa coefficient was used on 89 children in calculating inter-examiner consistency in diagnosing carious lesions. The kappa coefficients for the $\mathrm{dmft}$ and $\mathrm{dmfs}$ indices were 0.98 and 0.71 , respectively, whereas the kappa coefficient for the DMFT index was 0.77 .

\section{Statistical analyses}

SAS version 9.2 was used for analyzing the data. Differences between dependent (caries, plaque and tooth morphology) and independent (age, gender and school) variables were tested, using analysis of variance and $t$-test. The $\mathrm{d}_{2}$ - and $\mathrm{D}_{2}$-component consisted of caries scores $\mathrm{B}-\mathrm{E}$ and $1-4$, whereas the $\mathrm{d}_{3}$ - and $\mathrm{D}_{3}$-component consisted of caries scores C-E and 2-4, respectively.

\section{RESULTS}

\section{Disposition of subjects}

The total number of 7- to 8-year-olds examined was 1043 , of whom $54.7 \%$ were boys and $45.3 \%$ girls. The mean plaque score was 1.61 (s.d. $=0.51)$. The frequency distribution of plaque accumulation is shown in Table 3.

\section{Deciduous dentition}

The prevalence of dental caries in the deciduous dentition was $68.7 \%$. The mean $\mathrm{d}_{3} \mathrm{mft}$ and $\mathrm{d}_{3} \mathrm{mfs}$ scores were 2.8 and 5.0, respectively (Table 4). No gender effect was observed; the mean $\mathrm{d}_{3} \mathrm{mft}$ score for both boys and for girls was 2.7. The mean $\mathrm{d}_{3} \mathrm{mft}$ score of School 5 (3.7) was significantly higher than the mean $\mathrm{d}_{3} \mathrm{mft}$ score (2.6) of the other four schools $(P=0.0018)$. The $\mathrm{d}$-component constituted $75 \%$ of the $\mathrm{d}_{3} \mathrm{mft}$ index, while enamel carious lesions constituted $36 \%$ of the total number of carious lesions ( $\mathrm{d}_{2,3}$-component).

\section{Permanent dentition}

The prevalence of dental caries in the permanent dentition of these 7- to 8 -year-olds was $8.7 \%$. The mean $\mathrm{D}_{3} \mathrm{MFT}$ and $\mathrm{D}_{3} \mathrm{MFS}$ scores were 0.12 and 0.14 , respectively (Table 3 ). All cavitated dentine lesions were found

Table 3 Caries prevalence and caries experience scores in deciduous and permanent dentition using the DMFT/S index in 7-8-year-old children in Wuhan

\begin{tabular}{lccc}
\hline Parameters & T/t level, mean (s.d.) & S/s level, mean (s.d.) & Prevalence/\% \\
\hline$D_{2}$ & $0.55(1.08)$ & $0.75(1.63)$ & \\
$D_{3}$ & $0.11(0.42)$ & $0.13(0.53)$ & \\
$M$ & 0 & 0 & \\
$F$ & $0.01(0.11)$ & $0.01(0.11)$ & \\
$D_{2} M F$ & $0.56(1.08)$ & $0.75(1.63)$ & 27.3 \\
$D_{3} M F$ & $0.12(0.43)$ & $0.14(0.54)$ & 8.7 \\
$d_{2}$ & $3.24(3.06)$ & $5.07(5.97)$ & \\
$d_{3}$ & $2.06(2.50)$ & $3.41(5.23)$ & \\
$m$ & $0.16(0.52)$ & $0.42(1.39)$ & \\
$f$ & $0.58(1.23)$ & $1.20(2.67)$ & \\
$d_{2} m f$ & $3.99(3.43)$ & $6.69(6.91)$ & 78.9 \\
$d_{3} m f$ & $2.80(2.92)$ & $5.03(6.29)$ & 68.7 \\
\hline
\end{tabular}

s.d., standard deviation.

$D_{2} / d_{2}$ includes caries codes $1,2,3$ and $4 ; D_{3} / d_{3}$ includes caries codes 2,3 and 4 .

Table 4 Frequency distribution of children by caries experience $\left(\mathrm{d}_{3} \mathrm{mft} / \mathrm{D}_{3} \mathrm{MFT}\right)$

\begin{tabular}{lccccc}
\hline & \multicolumn{3}{c}{$\mathrm{d}_{3} \mathrm{mft}$} & & \multicolumn{2}{c}{$\mathrm{D}_{3} \mathrm{MFT}$} \\
\cline { 2 - 3 } \cline { 6 - 6 } Score & Frequency $(N)$ & Percentage/\% & & Frequency $(N)$ & Percentage/\% \\
\hline 0 & 327 & 31.4 & & 952 & 91.3 \\
1 & 139 & 13.3 & & 66 & 6.3 \\
2 & 114 & 10.9 & & 18 & 1.7 \\
3 & 110 & 10.6 & & 7 & 0.7 \\
4 & 72 & 6.9 & & 1 & 0.1 \\
\hline
\end{tabular}

$N$, number of children. 
Table 5 Frequency distribution of children by plaque scores

\begin{tabular}{lcc}
\hline Scores & Frequency $(N)$ & Percentage/\% \\
\hline 0 & 19 & 1.8 \\
1 & 321 & 30.8 \\
2 & 646 & 62.0 \\
3 & 56 & 5.4 \\
\hline
\end{tabular}

$N$, number of children.

Table 6 Frequency distribution of children by pit and fissure morphology

\begin{tabular}{lcc}
\hline Scores & Frequency $(N)$ & Percentage/\% \\
\hline 1 & 144 & 15.4 \\
2 & 543 & 58.1 \\
3 & 247 & 26.5 \\
\hline
\end{tabular}

$N$, number of children.

in occlusal surfaces of first permanent molars; $6 \%$ of children had one decayed molar and $2 \%$ had two decayed molars. No gender effect was observed for the mean $\mathrm{D}_{3} \mathrm{MFT}$ and $\mathrm{D}_{3} \mathrm{MFS}$ scores. The mean $\mathrm{D}_{2} \mathrm{MFT}$ (0.69) and mean $\mathrm{D}_{2} \mathrm{MFS}$ (0.95) scores for girls were statistically significantly higher than those for boys $\left(\mathrm{D}_{2} \mathrm{MFT}=0.44\right.$ and $\left.\mathrm{D}_{2} \mathrm{MFS}=0.58\right)$ ( $P=0.0002$ and 0.0004 , respectively). No school effect was observed.

\section{Plaque accumulation}

The frequency distribution of children to levels of plaque accumulation is presented in Table 5 . The prevalence of plaque in these children was $98.2 \%$. The prevalence of medium and severe accumulation of plaque was $67.4 \%$.

\section{Pit and fissure morphology}

The frequency distribution of children to the different levels of pits and fissures is presented in Table 6 , showing scores 2 and 3 to be prevalent in $84.6 \%$ of the molar teeth examined. The mean score of pit and fissure morphology was 1.98 (s.d. $=0.57$ ).

\section{DISCUSSION}

As the purchasing power of consumers is increasing, it is expected that the caries situation in Chinese children will deteriorate in the coming years. As only cross-sectional caries epidemiological studies have been conducted, it is not possible to identify secular trends in caries prevalence and severity among these children. The current epidemiological investigation was carried out for two reasons: (i) to provide the baseline for a mixed-longitudinal assessment of dental caries in young children; and (ii) to support a study assessing the cost effectiveness of composite resin sealants in comparison to atraumatic restorative treatment sealants using glass ionomer in first permanent molars. In order to facilitate logistics for the latter, a convenient sample was chosen from schools near the School of Stomatology. The decision has reduced the external validity of the former reason, but as no regular follow-up longitudinal caries epidemiological survey has been carried out in the country, the decision to choose a convenient sample is considered justified.

The quality of the data collected was high, considering the high kappa values for the inter-examiner consistency test done for the caries indices studied.

Considering that dental caries is an age-related disease, the mean $\mathrm{dmft}$ score (2.8) of the 7- to 8-year-olds in the present study appears to be in line with the mean dmft of 2.0 obtained for 5 -year-olds in urban areas of Hubei Province during the Third National Oral Health Survey of $2005 .^{10}$ However, the mean $\mathrm{dmft}$ scores in the present study are somewhat lower than the mean $\mathrm{dmft}$ scores (3.7) for 6-year-olds in Yichang City, Hubei Province, in 1997, ${ }^{15}$ and those for 5-year-olds (3.1) in Guangxi and Hubei Provinces in 2002. ${ }^{16}$ Given that these studies have been conducted using different diagnostic criteria in different communities, they do not provide reliable data for the assessment of an upward trend in carious lesion development in deciduous teeth over the last decade.

The prevalence of dental caries in these 7- to 8-year-olds is high. Particularly disturbance is the high proportion of identified untreated cavitated dentine lesions and the low proportion of subsequently placed restorations. These indicate a low treatment pattern due to a combination of reasons that include insufficient knowledge of oral care among the general population and reduced accessibility of dental care in the survey area. They also show that a preventive and educational school oral health program is much needed. In the present study, one of the five schools has participated for years in the annual school oral health education and prevention program organized by the School of Stomatology. Despite these oral health activities, the dental caries situation of the children in this school was not better than that in the other study schools. Why one of the five schools had significant worse mean dmft score than the other schools had is not known.

The prevalence and severity of dental caries in the permanent dentition of these 7 - to 8-year-olds was low. The severity was somewhat lower than the mean DMFT score of 0.4 for 6-year-olds in 1995 in Wuhan.

The number of permanent first molars with enamel carious lesions and those with dentine carious lesions were found to be powerful caries predictors over a period of 4 years in a similar age group in Wuhan. ${ }^{11,17}$ That in the present study all cavitated dentine lesions were in the occlusal surface of first permanent molars which had only recently erupted, that a large proportion of occlusal surfaces in such teeth had enamel carious lesions and that the prevalence of dentine carious lesions in the primary molars was high, call for preventive action, particularly for the placement of sealants in high caries risk children. The fact that $26 \%$ of first permanent molars exhibited a deep pit and fissure pattern only supports the call for action.

As caries is a socioeconomic related disease, a questionnaire was designed to acquire the oral health habit, education and economic background of children. However, as the children are too young to give an accurate answer, $40 \%$ drop-off of the questionnaire make the analysis impossible. This information will be collected again 2 years later and reported in future. Although the background is not very clear yet, four of the schools are associated primary school of the university, which means the staffs of the university have advantages to choose it. Meanwhile, five schools are located in the centre of Wuhan City. This implies that the children are from medium- to high-income family with low- to high-education background.

In conclusion, the prevalence of dental caries in the deciduous and permanent dentitions of 7- to 8-year-old children was high. Deep pits and fissures in high caries risk children should be sealed. Appropriate preventive measures should be instituted.

\section{ACKNOWLEDGEMENTS}

The authors are grateful to Dr Li-Hua Liu for examining the children and to Dr Rong Wang, Dr Te Zhou and Ms Ting Peng for recording data.

1 Zhou Y, Yang JY, Lo EC et al. The contribution of life course determinants to early childhood caries: a 2-year cohort study. Caries Res 2012; 46(2): 87-94. 
2 Frencken JE, van't Hof MA, Truin GJ et al. Cohort effects in the prevalence of caries in child populations in Tanzania. J Dent Res 1989; 68(12): 1777-1780.

3 Ahovuo-Saloranta A, Hiiri A, Nordblad A et al. Pit and fissure sealants for preventing dental decay in the permanent teeth of children and adolescents. Cochrane Database Syst Rev 2008; 8(4): CD001830.

4 Zhang Q, van Palenstein Helderman WH, van't Hof MA, Truin GJ. Chlorhexidine varnish for preventing dental caries in children, adolescents and young adults: a systematic review. Eur J Oral Sci 2006; 114(6): 449-455.

5 Azarpazhooh A, Main PA. Pit and fissure sealants in the prevention of dental caries in children and adolescents: a systematic review. J Can Dent Assoc 2008; 74(2): $171-177$.

6 Simonsen RJ. Glass ionomer as fissure sealant-a critical review. J Public Health Dent 1996; 56(3): 146-149.

7 Beiruti N, Frencken JE, van't Hof MA et al. Caries preventive effect of resin-based and glass ionomer sealants over time: a systematic review. Community Dent Oral Epidemiol 2006; 34(6): 403-409.

8 Yengopal V, Mickenautsch S, Bezerra AC et al. Caries-preventive effect of glass ionomer and resin-based fissure sealants on permanent teeth: a meta analysis. J Oral Sci 2009; 51(6): 373-382.

9 Wang HY, Petersen PE, Bian JY et al. The second national survey of oral health status of children and adults in China. Int Dent J 2002; 4(6): 283-290.

10 Tai BJ, Jiang H, Du MQ et al. The investigation report of caries prevalence in Hubei Province. J Oral Sci Res 2007; 23(3): 223-225
11 Zhang $Q$, van Palenstein Helderman WH. Caries experience variables as indicators in caries risk assessment in 6-7-year-old Chinese children. J Dent 2006; 34(9): 676-681.

12 Cheng RB, Tao W, Zhang $Y$ et al. Analysis of the first permanent molar caries epidemiological investigation in area of northeast China. Hua Xi Kou Qiang Yi Xue Za Zhi 2008; 26(1): 73-76. Chinese.

13 Greene JC, Vermillion JR. The simplified oral hygiene index. J Am Dent Assoc 1964; 68(1): 7-13.

14 Symons AL, Chu CY, Meyers IA. The effect of fissure morphology and pretreatment of the enamel surface on penetration and adhesion of fissure sealants. J Oral Rehabil 1996; 23(12): 791-798.

15 Tai BJ, Petersen PE, Jing $\mathrm{H}$ et al. Dental caries and oral health behavior of 6-year-old schoolchildren in Yichang City, PR China. Chin J Dent Res 2003; 6(1): 5-10.

16 Du M, Luo $Y$, Zeng $X$ et al. Caries in preschool children and its risk factors in 2 provinces in China. Quintessence Int 2007; 238(2): 143-151.

17 Petersen PE, Guang LX. Dental caries prevalence in a group of school children in Wuhan City, PR China. Community Dent Oral Epidemiol 1994; 22(6): 465-466.

(c) This work is licensed under a Creative Commons Attribution-NonCommercial-NoDerivative Works 3.0 Unported License. To view a copy of this license, visit http:// creativecommons.org/licenses/by-nc-nd/3.0 